\title{
The potential anti-inflammatory role of adiponectin in food allergy: a case-control study on children
}

\author{
Mahshid Sirjani ${ }^{1}$, Raheleh Shokouhi Shoormasti ${ }^{1}$, Nastaran Sabetkish ${ }^{1,2}$, Maryam Ayazi ${ }^{1}$, Nazanin \\ Khodayari Namini ${ }^{1}$, Behnoosh Tayebi ${ }^{1}$ and Zahra Pourpak ${ }^{1 *}$ \\ ${ }^{1}$ Immunology, Asthma and Allergy Research Institute, Tehran University of Medical Sciences, Tehran 1419733151, Iran \\ ${ }^{2}$ Pediatric Urology and Regenerative Medicine Research Center, Section of Tissue Engineering and Stem Cells Therapy, Tehran \\ University of Medical Sciences, Tehran 1419433151, Iran
}

(Submitted 16 April 2018 - Final revision received 8 August 2018 - Accepted 13 August 2018)

\section{Abstract}

We aimed to assess the possible relationship between food allergy and two key adipokines - leptin and adiponectin - in children with food allergy. A total of forty patients with definite diagnosis of food allergy according to clinical history and specific IgE (sIgE) for food allergens (group I) were enrolled in this pilot study. The control group (group II) included thirty children with no evidence of allergic symptoms. Serum levels of leptin and adiponectin were measured by ELISA. Meanwhile, sIgE was measured for the eight most common food allergens by the immunoblot method in all participants. The median ages in groups I and II were 18.5 and 23.5 months, respectively. The respective Caesarean section rate was 64.9 and $16.7 \%$ in groups I and II $(P<0.001)$. Serum levels of adiponectin were significantly higher in the patient group compared with controls (24.11 (sD 12.14) $v .10 \cdot 67$ (sD 12.23) $\mu \mathrm{g} / \mathrm{ml}, P<0.001$ ), whereas no statistically meaningful difference was detected in serum leptin concentrations $(P=0.92)$. There was a significant inverse relationship between age and adiponectin levels in group I $(P=0.002$, $r-0.479)$ and group II $(P=0.04, r-0.365)$, and it was more significant in group I. The most common allergens in the patient group were wheat (52.5\%), hazelnut (52.5\%), cow's milk (50\%) and egg white (30\%). The results of this study suggest an essential link between adiponectin and food allergy that is probably unlikely to be affected by obesity as a confounding factor.

Key words: Leptin: Adiponectin: Immunoglobulin E: Food allergies

The prevalence of immediate hypersensitivity reactions to food, commonly called food allergies, is on the rise at an alarming rate in industrialised countries ${ }^{(1)}$, although the reasons are not clear $^{(2)}$. The increase in the incidence of food allergy is likely to be related to environmental factors because its rise is more rapid than genetic deviation ${ }^{(3)}$. Factors such as hygiene and lack of exposure to pathogenic microbes, the composition of the intestinal microbiota, diet, obesity, vitamin D insufficiency/ deficiency and environmental chemical exposure have all been proposed to contribute to this alarming rise in the rate of food allergy in countries with a Westernised lifestyle ${ }^{(2,4,5-7)}$.

Recent studies revealed that both obesity and allergy had simultaneously become more prevalent throughout the world. This has brought the hypothesis that there is a relationship between these two chronic diseases ${ }^{(5)}$. Obesity and immune system are linked with the adipose tissue ${ }^{(8)}$. The chronic systemic inflammation, induced by excess adipose tissue ${ }^{(9)}$, may explain the relationship between obesity and allergy ${ }^{(10)}$. However, there are inconsistencies in research results. Despite the association between obesity and the development of atopic dermatitis among children ${ }^{(5)}$, no clear association was found between obesity and the prevalence of other allergic diseases such as allergic rhinitis and allergic conjunctivitis. The results of NHANES from 2005 to 2006 revealed that systemic inflammation may be considered as a contributor in the development of allergic diseases, and obesity may increase the prevalence of allergic diseases, mainly food allergy ${ }^{(10)}$.

The aim of this study was therefore to investigate whether adipokines as markers of obesity and fat tissue are associated with food allergy. Despite the existence of other adipokines, we decided to evaluate the role of adiponectin and leptin as the most fundamental ones in this pilot study.

\section{Methods}

The methodology of this study was conducted according to the Declaration of Helsinki and approved in research and ethical committee of Immunology, Asthma and Allergy Research Institute (IAARI) (IR.TUMS.IAARI.REC.1395.385). The statistical

Abbreviation: $\operatorname{sigE}$, specific IgE.

* Corresponding author: Z. Pourpak, fax +98 21 6642899, email pourpakz@sina.tums.ac.ir 
power of the study was $80 \%$, and two-sided significant level was $0 \cdot 05$. The formula for determining the sample size is mentioned below:

$$
n=\frac{\left(\frac{1}{q 1}+\frac{1}{q 0}\right)(Z \alpha+Z \beta)}{\left(\frac{E}{S}\right) 2}
$$

where $q 1=0.55, q 0=0.45, Z \alpha=1.95996, Z \beta=0.84162$, standardised effect size $=(E / S)=0 \cdot 65$, case group $=42$ and control group $=34$.

Accordingly, forty patients with food allergy (group I) were selected from patients referred to IAARI for allergy diagnosis from December 2014 to April 2015. Diagnostic criteria for food allergy included a clinical history of allergic reaction to food and a positive specific IgE (sIgE) test to food allergens. The level of sIgE was determined for all children with typical symptoms of food allergy. The diagnosis was confirmed with $\operatorname{sigE}>0.7 \mathrm{IU} /$ $\mathrm{ml}$, independently of type of food allergens as a pilot study with limited patients. Then, children with typical symptoms along with positive laboratory findings were included in this study ${ }^{(11)}$. Considering the fact that DHA and EPA supplementations may result in increased adiponectin level, all children who had received any kind of dietary modification and supplementation were excluded from this study.

In all, thirty children with no allergy symptoms who were referred to the Urology Department of Children Medical Center for minor surgical procedures (such as circumcision, hypospadias or hernia) were selected as controls (group II). Case and controls were matched for age and sex. After obtaining informed consent, a data gathering questionnaire (including demographic and clinical data) was completed for both groups. To monitor the growth, weight-for-age and BMI-for-age percentiles were calculated for subjects under and above 2 years of age, respectively. Subsequently, $2-3 \mathrm{ml}$ of blood sample was collected from the remaining samples taken for allergic diagnosis or routine tests for surgical procedures in study groups. Their serums were separated and stored at $-20^{\circ} \mathrm{C}$. Serum sIgE levels were measured using RIDA qline Allergy kit (R-Biopharm) in both groups. The RIDA qline allergy test is an immunoassay technique on nitrocellulose membrane. Allergens were coated on the surface of the membrane. The strips were evaluated with a 3D scanner and qline software. The unit of SIgE is $\mathrm{IU} / \mathrm{ml}$. In addition to $\mathrm{sIgE}$ measurements, serum leptin

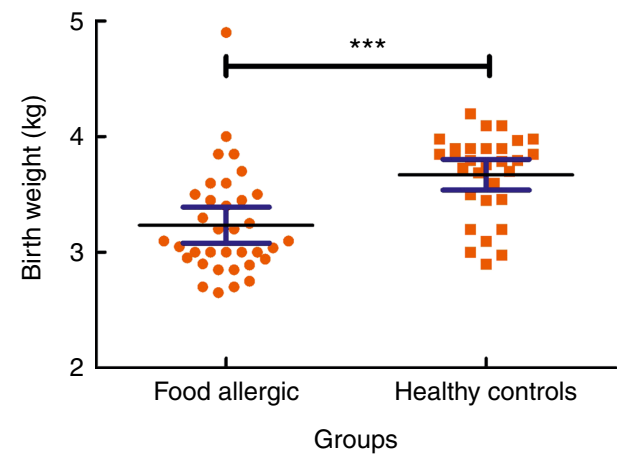

and adiponectin levels were assessed by sandwich ELISA kit of leptin (LDN) and adiponectin (R\&D Systems).

\section{Statistical analysis}

Data analysis was performed using IBM SPSS 20. The frequency and percentage of categorical data were determined. Parametric and non-parametric statistical tests were selected considering quantitative data distribution (using Kolmogorov-Smirnov test). Independent $t$ test and Mann-Whitney were applied to compare a quantitative variable between two independent groups. Pearson's and Spearman's tests were used to evaluate the correlation between two quantitative groups. A $P$-value $<0.05$ was considered significant.

\section{Results}

The frequency of skin (eczema, urticaria, itching), respiratory (sneezing, dyspnoea, wheezing, cough) and gastrointestinal (abdominal pain, diarrhoea, vomiting, blood in stool, nausea, reflux, constipation) symptoms were 82.5, 45 and $70 \%$, respectively. On the basis of clinical history, 25 and $15 \%$ of patients reported anaphylaxis and angio-oedema-like symptoms that were not confirmed by laboratory findings. The median ages of participants in patient and control groups were 18.5 $(Q 1=8, Q 3=42)$ and $23.5(Q 1=18, Q 3=28)$ months, respectively. The results of this study revealed that there was no significant difference in sex and age between the study groups $(P>0.05)$. However, there was a disproportionate representation of males in groups I and II (28/12 and 19/11, respectively). Normal vaginal delivery rates were significantly lower in the patient group (21.6 v. 56.7\%, $P=0.003)$. Elective Caesarean section rate in groups I and II was in turn 64.9 and $16.7 \%$, respectively, showing a significant difference among groups $(P<0.001)$. There was no significant difference in breastfeeding and bottle-feeding rates between two groups. As depicted in Fig. 1, a significant difference was detected between groups I and II in birth weight (3.23 (SD 0.45)v.3.67 (SD 0.35) kg, $P<0.001)$ and birth length (0.49 (SD 0.02) v. 0.51 (SD 0.01) $\mathrm{m}$, $P=0.002$ ). According to the growth rate indices, neither the patient nor the control groups were overweight or obese. 
Serum levels of adiponectin were significantly higher in the patient group compared with the controls (24.11 (SD 12.14) $v$. 10.67 (sD 12.23$) \mu \mathrm{g} / \mathrm{ml}, P<0.001)$. The median serum leptin concentration was $2.15(Q 1=1.35, Q 3=3.85)$ in group I, whereas the median level of this mediator was $2 \cdot 35(Q 1=1 \cdot 21$, $Q 3=5.01)$ in group II, with no statistically significant difference among groups $(P=0.92)$ (Fig. 2). There was a significant inverse relationship between age and adiponectin levels in group I $(P=0.002, r-0.479)$ and group II $(P=0.04, r-0.365)$. However, the results were more significant in group I. In spite of a positive correlation between age and leptin levels in group I, the difference was not statistically significant $(P=0.053$, $r$ 0.308). There was no statistically significant difference between the level of different adipokines and type of delivery. Similarly, no statistically significant difference was detected between the level of adipokines and sexes.

On the basis of sIgE levels, the most common allergens in the patient group were wheat $(52.5 \%)$, hazelnut $(52.5 \%)$, cow's milk ( $50 \%$ ), bovine serum albumin (BSA, 36.7\%) and egg white (30\%) (Fig. 3). The concentrations of adipokines (leptin and adiponectin) were compared between patients according to sensitisation (positive or negative) to milk, wheat, hazelnut, peanut, egg white, egg yolk, BSA and carrot. Accordingly, a significant difference was detected between the concentration of adiponectin and positive sIgE to carrot allergen $(P=0.004)$, as well as adiponectin concentration and sIgE to BSA allergen $(P=0 \cdot 01)$. No significant correlation was detected between the sum of sIgE level (all evaluated allergens) and none of the measured adipokines.

\section{Discussion}

Food allergy is considered a complex genetic disorder in which environmental factors have robust influence, resulting in a recent increase in its prevalence around the world ${ }^{(12)}$. One of the most important environmental factors is obesity ${ }^{(7)}$. Growing evidence has focused on the role of obesity and the adipose tissue on the immune system ${ }^{(8)}$. Indeed, secretion of a variety of protein signals such as leptin and adiponectin from adipocytes has caused the white adipose tissue to be considered as an important endocrine organ ${ }^{(4)}$. The adipokines are involved in the pathways linking obesity and allergic sensitisation ${ }^{(13)}$. As food allergies usually begin in children aged below 5 years and at this age period detection of obesity is not routine, in this study we investigated obesity and being overweight through the pivotal adipose tissue markers such as adiponectin and leptin. According to our findings, the serum level of adiponectin was significantly higher in the case group compared with the control group. However, no statistically significant difference was detected in serum leptin concentrations between the study groups. These results are in contrats to the study of Radon et $a l^{(13)}$, which was conducted in an adult population. In adults, it seems that obesity can induce an imbalance in the immune cell responses and may decrease the body's immunological tolerance ${ }^{(5)}$. In infant studies, there is no evidence of obesity's influence on food allergy ${ }^{(10,14)}$. Adiponectin has antiinflammatory action and is capable of attenuating allergen responses ${ }^{(13)}$. Higher adiponectin level in allergic patients of the current study may be due to lower birth weight and subsequently lower fat tissue in this group compared with healthy controls. It seems that lower pregnancy termination age owing to a higher rate of Caesarean sections in group I could result in lower birth weight and length in this group. As adiponectin has anti-inflammatory action, it seems reasonable to have higher adiponectin levels in allergic children owing to inflammatory conditions in comparison with controls with no inflammatory responses. This increase is unlikely to be affected by obesity as a confounding factor. In fact, these children are less likely to be

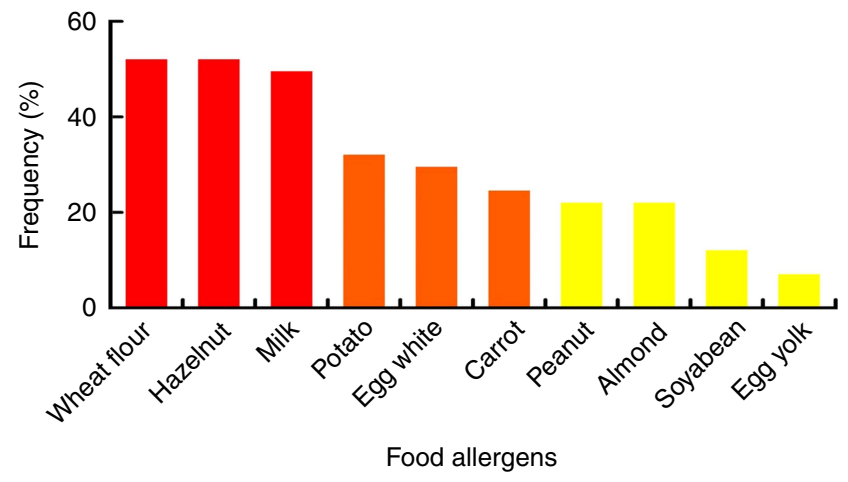

Fig. 3. Prevalence of the most common allergens in the patient group.
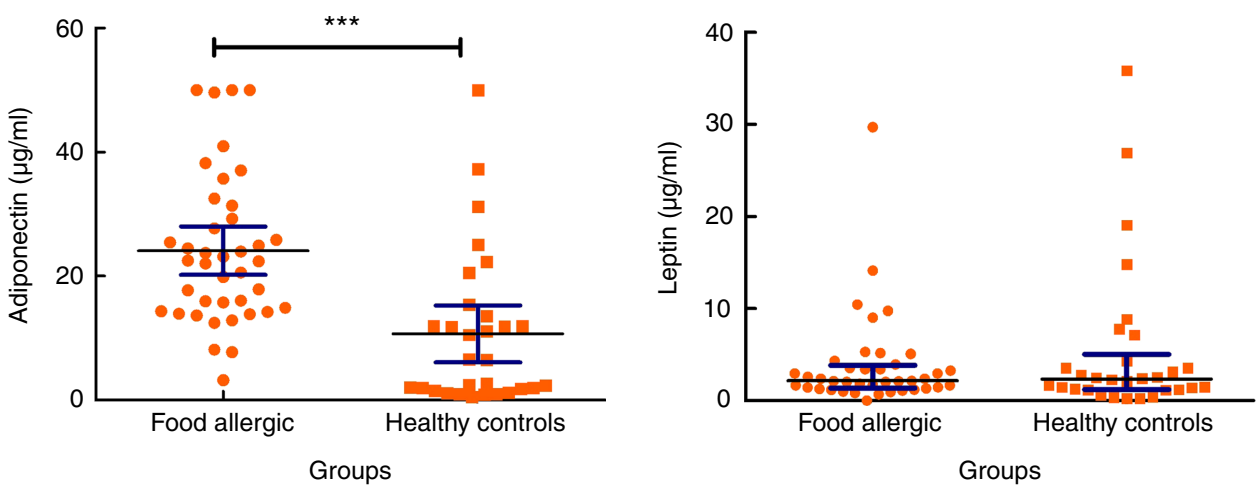

Fig. 2. Serum levels of adiponectin and leptin in allergic patients and healthy controls. IQR, interquartile range. For adiponectin, values are means and $95 \%$ confidence intervals. For leptin, values are medians and interquartile ranges. ${ }^{\star \star \star} P<0.001$. 
overweight owing to dietary restrictions and natural food refusal of children to avoid unpleasant digestive and dermatological symptoms.

The result indicated male predominance in both case and control groups. Because of the small sample size in our study, the pattern of sex disparity in food allergy is independent of the specific type of allergenic food. Nevertheless, there is a significantly higher male:female ratio in group I. Our data are comparable to the presence of sex disparity in the published studies in food allergy in PubMed database ${ }^{(15)}$. The immunemodulating effect of sex hormone could provide a convenient explanation for food allergy after puberty, more than that provided by our finding during infancy and childhood. Genetic predisposition could explain the increased complaints of food allergy among prepubescent boys in our analysis. The sexual genotype is the basis of sex differences, and an X-linked recessive trait associated with allergic disease would be more likely to be unmasked in males, being able to explain the predominance of food allergies in males at a very young age $e^{(15)}$.

Our findings revealed that delivery by Caesarean section was significantly higher in the case group compared with controls. This result is compatible with other studies regarding the association between Caesarean section and allergic outcome. The first colonisation of the neonatal gut has occurred through the process of vaginal delivery with maternal vaginal bacteria, whereas Caesarean section babies are deprived of this natural exposure and exhibit a different gut flora ${ }^{(16,17)}$. This different composition of the flora could interact with the normal GI immunological tolerance and could be an explanation for the association between higher cesarean section rates in food-allergic patients. According to the relationship of type of delivery and development of immune system $^{(18)}$, controlling the incidence of elective Caesarean sections may have a positive impact in reducing the prevalence of allergic diseases including food allergy.

The results of our previous study on non-fatal anaphylactic attacks among sixty-three Iranian children revealed that the most common causes of anaphylactic reactions were milk and wheat ${ }^{(19)}$. In another study, milk, egg and wheat were determined to be the most common food allergens among 313 Iranian allergic children ${ }^{(20)}$. The results of current study were compatible with our previous findings in terms of common food allergens among atopic children. Accordingly, wheat, hazelnut, cow's milk and egg white were found to be the most common food allergens.

\section{Conclusion}

Considering the fact that adiponectin level was significantly higher in the patient group and this level had an inverse relationship with age of the patients, it may be concluded that the anti-inflammatory characteristics of this cytokine may result in symptom improvement in older atopic children. In other words, atopic paediatrics may produce higher level of this antiinflammatory protein to deal with inflammatory reactions. Therefore, it seems that increased level of adiponectin in allergic patients, regardless of weight changes, may be attributed to the anti-inflammatory characteristics of this protein. However, this still remains as a hypothesis that needs further investigations to be confirmed. Larger group of allergic children and more precise evaluations on other defined adipokines other than adiponectin and leptin are required to draw firm conclusions and find the role of each adipokine in the allergy pathway.

\section{Acknowledgements}

We highly acknowledge the Immunology, Asthma and Allergy Research Institute/Tehran University of Medical Sciences for supporting this work (grant no. 95-02-40-31596).

M. S. contributed to conception and design, acquisition and interpretation and drafted the manuscript. R. S. S. contributed to analysis and interpretation and drafted the manuscript. N. S. contributed to acquisition and interpretation, and critically revised the manuscript. M. A., N. K. N. and B. T. contributed to acquisition and critically revised the manuscript. Z. P. contributed to conception or design, acquisition and interpretation and critically revised the manuscript. All authors gave final approval and agree to be accountable for all aspects of work, ensuring integrity and accuracy.

The authors declare that there are no conflicts of interest.

\section{References}

1. Tang ML \& Mullins RJ (2017) Food allergy: is prevalence increasing. Intern Med J 47, 256-261.

2. Benede S, Blazquez AB, Chiang D, et al. (2016) The rise of food allergy: environmental factors and emerging treatments. EBioMedicine 7, 27-34.

3. Metcalfe DD, Sampson HA, Simon RA, et al. (editors) (2014) Food Allergy Adverse Reaction to Foods and Food Additives, 5th ed. Hoboken, NJ: Wiley-Blackwell.

4. Hersoug LG \& Linneberg A (2007) The link between the epidemics of obesity and allergic diseases: does obesity induce decreased immune tolerance. Allergy 62, 1205-1213.

5. Baumann S \& Lorentz A (2013) Obesity - a promoter of allergy Int Arch Allergy Immunol 162, 205-213.

6. Manzel A, Muller DN, Hafler DA, et al. (2014) Role of "Western diet" in inflammatory autoimmune diseases. Curr Allergy Asthma Rep 14, 404.

7. Sicherer SH \& Sampson HA (2018) Food allergy: a review and update on epidemiology, pathogenesis, diagnosis, prevention, and management. $J$ Allergy Clin Immunol 141, 41-58.

8. de Heredia FP, Gomez-Martinez S \& Marcos A (2012) Obesity, inflammation and the immune system. Proc Nutr Soc 71, 332-338.

9. Federico A, D'Aiuto E, Borriello F, et al. (2010) Fat: a matter of disturbance for the immune system. World J Gastroenterol 16 , 4762-4772.

10. Visness CM, London SJ, Daniels JL, et al. (2009) Association of obesity with IgE levels and allergy symptoms in children and adolescents: results from the National Health and Nutrition Examination Survey 2005-2006. J Allergy Clin Immunol 123, 1163-1169; 1169.e1161-1164.

11. Hwang H, Kwon J, Kim JY, et al. (2016) The RIDA allergy screen versus the phadiatop test in 430 consecutive patient specimens. Lab Med 47, 20-29.

12. Grundy J, Matthews S, Bateman B, et al. (2002) Rising prevalence of allergy to peanut in children: data from 2 sequential cohorts. J Allergy Clin Immunol 110, 784-789. 
13. Radon K, Schulze A, Schierl R, et al. (2008) Serum leptin and adiponectin levels and their association with allergic sensitization. Allergy 63, 1448-1454.

14. Eldin LB, Algamal HA, El-Dory GF, et al. (2008) Relation between obesity, lipid profile, leptin and atopic disorders in children. Egypt J Pediatr Allergy Immunol 6, 27-34.

15. Kelly C \& Gangur V (2009) Sex disparity in food allergy: evidence from the PubMed database. J Allergy (Cairo) 2009, 159845.

16. Bager P, Wohlfahrt J \& Westergaard T (2008) Caesarean delivery and risk of atopy and allergic disesase: meta-analyses. Clin Exp Allergy 38, 634-642.
17. Eggesbø M, Botten G, Stigum H, et al. (2003) Is delivery by cesarean section a risk factor for food allergy. J Allergy Clin Immunol 112, 420-426.

18. Neu J \& Rushing J (2011) Cesarean versus vaginal delivery: long-term infant outcomes and the hygiene hypothesis. Clin Perinatol 38, 321-331.

19. Barzegar S, Rosita A, Pourpak Z, et al. (2010) Common causes of anaphylaxis in children: the first report of anaphylaxis registry in iran. World Allergy Organ J 3, 9-13.

20. Hosseini S, Shoormasti RS, Akramian R, et al. (2014) Skin prick test reactivity to common aero and food allergens among children with allergy. Iranian J Med Sci 39, 29-35. 